\title{
Modeling of a CMOS Convective Accelerometer for HDL integration
}

\author{
O. Leman, A. Chaehoi, F. Mailly, L. Latorre and P. Nouet \\ Laboratoire d'Informatique de Robotique et de Microélectronique de Montpellier \\ UMR 5506 - CNRS / University Montpellier II, 161 rue Ada, 34392, \\ Montpellier - France \\ latorre@lirmm.fr
}

\begin{abstract}
This paper introduces an analytical modeling of a convection heat CMOS accelerometer. The modeling approach relies on the use of fundamental results and is validated using both experimental data issued from test vehicles and FEM analysis. Since such accelerometers are based on the CMOS technology, the proposed model is intended for further HDL integration. The objective is to enable system level simulation and optimization within standard microelectronic CAD software.
\end{abstract}

\section{INTRODUCTION}

Convective accelerometers are quite different from their seismic-mass-based equivalents. Their working principle relies on the heating of a small volume of gas, air at atmospheric pressure in our case, which is disturbed by an applied acceleration. As a result, a temperature gradient appears in the gas volume, which can be measured by means of temperature sensors. Such structures have been investigated for decades and recently reported on silicon $[1,2,3]$. Several studies have been carried-out on this kind of sensors using $\mathrm{Pt}$ resistors both as heating and sensing elements [4].

This work addresses the implementation of this sensing principle on standard CMOS technology using the "Front Side Bulk Micromachining" technique to release mechanical structures. This monolithic approach allows batch production and dramatic costs reductions over hybrid sensors which use MEMS and signal conditioning on several dedicated dies.

Monolithic CMOS thermal accelerometers have been developed and commercialized using thermocouples as temperature-sensing elements [5]. In this paper, the sensing elements are polysilicon resistors from the CMOS technology, which feature a linear temperature response. The aim of such work is to provide microelectronics engineers with MEMS IPs, similar in use to analog cells coming from design kit libraries, which can be tailored to the desired dimensions and integrated among the signal conditioning electronics, and then simulated together to validate the whole system.

Our modeling approach relies on the use of heat transfer fundamentals, in association with empirical parameters. Experimental data is provided by both FEM simulations and the characterization of physical test vehicles. Those complementary methods are efficient to handle the problem complexity in terms of both physical phenomena and geometry while leading to a compact model, ready for HDL integration and device optimization.

\section{DEVICE UNDER STUDY}

The accelerometer is composed of three thin bridges suspended over a cavity (i.e. thermally isolated from the substrate) and oriented $45^{\circ}$ from the cavity boundaries in order to speed-up the etching process (see Fig. 1 and Fig. 2). The bridges are composed of various materials, as usually found in the CMOS back-end layers (oxide, polysilicon, aluminum, and nitride). In particular, polysilicon is used to implement resistors, for both heating $(\mathrm{Rh})$ and sensing $\left(\mathrm{Rd}_{1}\right.$, $\mathrm{Rd}_{2}$ ) purposes.

The heater $R_{H}$ is supplied by an electrical current in order to create a temperature gradient in the top and bottom cavities: the temperature is then maximum at the heater location and minimum at the cavities boundaries. In absence of acceleration, the detectors measure the same temperature for symmetry reasons. As a result of an acceleration along the $\mathrm{x}$-axis, the temperature gradient deforms and a temperature difference can be measured across the detectors. Because the resistors exhibit significant temperature dependence, the thermal signal is easily converted into a voltage by means of a simple conditioning circuit (e.g. a Wheatstone bridge). 


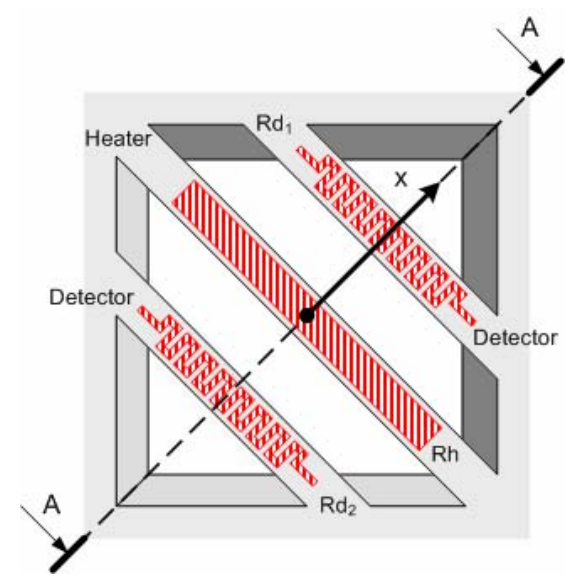

(a)

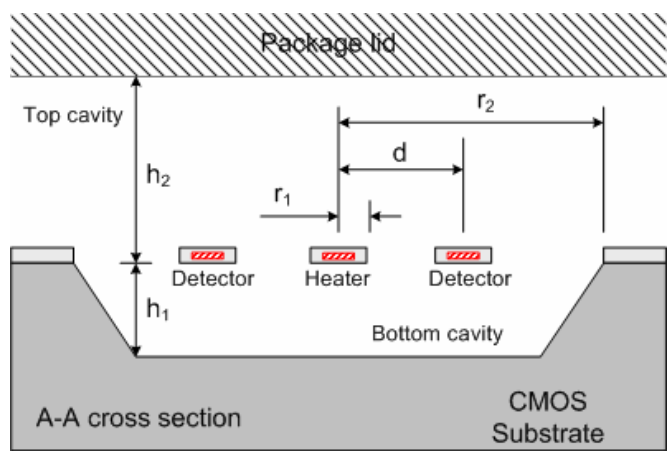

(b)

Figure 1. (a) top view of the sensor under study. (b) Cross section of the sensor with dimension parameters.

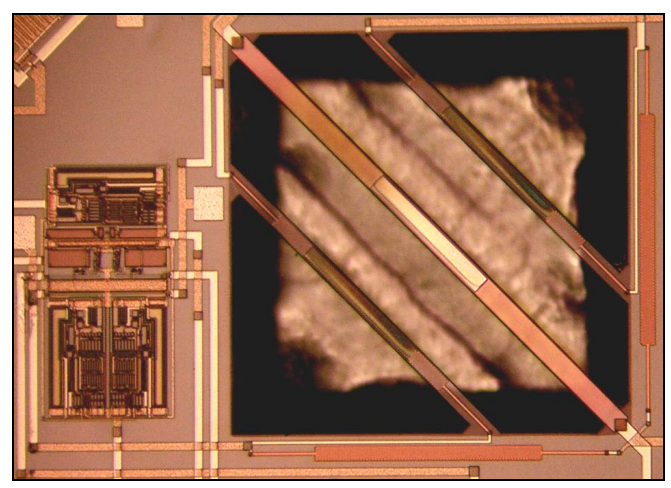

\begin{tabular}{|c|c|c|}
\hline & $\mathrm{A}$ & $\mathrm{B}$ \\
\hline $\mathrm{r}_{1}$ & $20 \mu \mathrm{m}$ & $20 \mu \mathrm{m}$ \\
\hline $\mathrm{r}_{2}$ & $570 \mu \mathrm{m}$ & $340 \mu \mathrm{m}$ \\
\hline $\mathrm{d}$ & $200 \mu \mathrm{m}$ & $125 \mu \mathrm{m}$ \\
\hline $\mathrm{Rh}$ & $720 \Omega$ & $350 \Omega$ \\
\hline $\mathrm{Rd}_{1}, \mathrm{Rd}_{2}$ & $50 \mathrm{k} \Omega$ & $50 \mathrm{k} \Omega$ \\
\hline $\mathrm{h}_{1}$ & \multicolumn{2}{|c|}{$\approx 300 \mu \mathrm{m}$} \\
\hline $\mathrm{h}_{2}$ & \multicolumn{2}{|c|}{$\approx 1 \mathrm{~mm}$} \\
\hline
\end{tabular}

Figure 2. Picture of the test vehicle and associated physical parameters

\section{MODELING}

The proposed model is represented in Fig. 3. Section 1 models the heating source. Heat transfer in the air, from the heater to the detectors, is then separated into two functional blocs: section 2 describes the conduction transfer, which essentially rules the common mode on detectors, while section 3 expresses convective transfer from which depends the output signal. Finally, section 4 implements detectors transfer function.

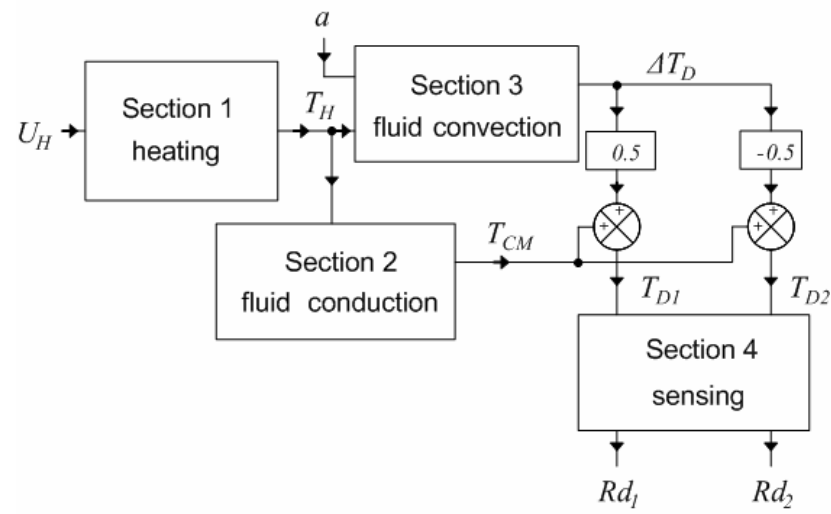

Figure 3. Functionnal bloc representation of the model

\section{A. Section 1: Heating Source}

During normal operation, the heating bridge is supplied by a dc current in order to set an initial temperature distribution in the cavity. Therefore, only a static representation of the heating bridge is required for the simulation of normal operation.

The electrical power supplied to the heating resistor $\left(P_{H}\right)$ is dissipated by heat transfer toward the fluid (conduction \& convection) and also toward the substrate (conduction in the beam). Conduction in the beam leads to a parabolic temperature profile along the beam with a maximum temperature in the middle of the bridge. For system level simulation, such exactitude is however not required and the model implements a simplified expression considering an average temperature for the heater, given by:

$\Delta T_{H}=T_{H}-T_{A}=R_{t h} \times P_{H}$

where $T_{H}$ and $T_{A}(\mathrm{~K})$ are respectively the heater temperature and ambient temperature and $R_{t h}(\mathrm{~K} / \mathrm{W})$ corresponds to an equivalent thermal resistance of the beam.

The linear relationship between the electrical power and the heater temperature has been verified experimentally on the two test vehicles A and B (Fig. 4) providing empirical data for $R_{t h}$ in both cases $\left(R_{t h}(A)=10800 \mathrm{~K} / \mathrm{W}\right.$, $\left.R_{t h}(B)=15200 \mathrm{~K} / \mathrm{W}\right)$. Note that the temperature is calculated from the measured heater resistance using a TCR of $0.09 \% /{ }^{\circ} \mathrm{C}$ (given by the foundry). Also note that both heaters have been destroyed beyond $700{ }^{\circ} \mathrm{C}$. 


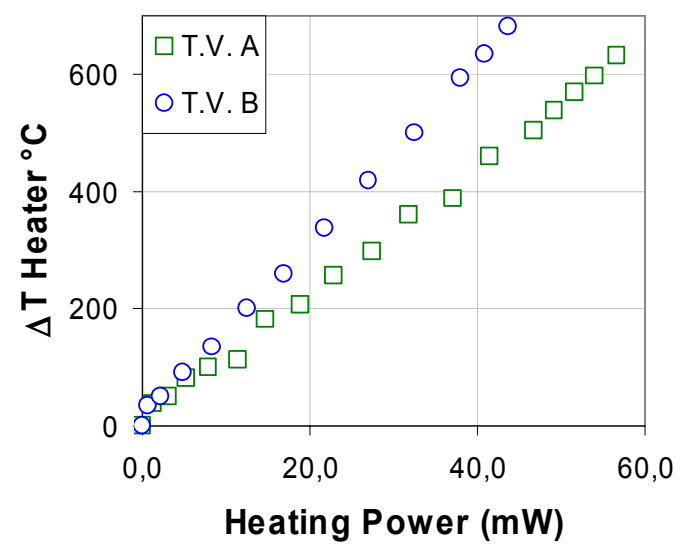

Figure 4. Characterization of the heater temperature as a function of the electrical power supplied to the resistor.

Finally, the resistance value of the heater is fed back into the model as a function of the heater temperature using the polysilicon $T C R$.

\section{B. Section 2: Fluid Conduction}

This functional bloc implements the heat conduction in the air, which rules the initial temperature of both detectors (i.e. the temperature common mode). Preliminary analyses have been carried out with ANSYS using a simple 2D model of the device cross section (Fig. 1.b). Then an analytical model is proposed based on a cylindrical geometry approximation.

In Fig. 1, $r_{1}$ and $r_{2}$ can be used as characteristic dimensions for the MEMS. Assuming a radial heat flow and a gas behavior equivalent to the one of a solid, (i.e. no movement), the temperature $\mathrm{T}$ of a point at distance $\mathrm{r}$ from the heater is ruled by:

$\frac{d}{d r}\left(r \lambda(t) \frac{d T}{d r}\right)=0$ and $\lambda(t)=\lambda_{0}(1+\gamma T)$

With, $\lambda_{0}=6.84 \cdot 10^{-3} \mathrm{~W} \cdot \mathrm{m}^{-1} \cdot \mathrm{K}^{-1}$ the air conductivity at $0^{\circ} \mathrm{K}$ and $\gamma=9.73 .10^{-3} \mathrm{~K}^{-1}$ the air coefficient of thermal variation of conductivity at $0^{\circ} \mathrm{K}$.

Separating the variables, we obtain after integration:

$\lambda_{0}\left(T+\gamma \frac{T^{2}}{2}\right)=A \ln (r)+B$

Constants $\mathrm{A}$ and $\mathrm{B}$ are determined thanks to boundary condition: $T=T_{H}$ for $r=r_{1}$ and $T=T_{A}$ for $r=r_{2}$. We finally obtain:

$$
T+\gamma \frac{T^{2}}{2}=\frac{\left[\left(T_{H}-T_{A}\right)+\frac{\gamma}{2}\left(T_{H}{ }^{2}-T_{A}{ }^{2}\right)\right]}{\ln \left(\frac{r_{2}}{r_{1}}\right)} \cdot \ln \left(\frac{r}{r_{1}}\right)+T_{H}+\gamma \frac{T_{H}{ }^{2}}{2}
$$

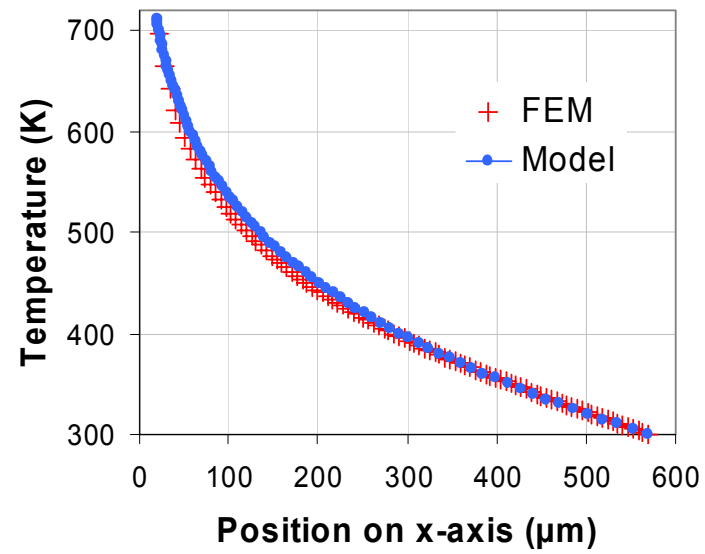

Figure 5. Temperature profile along $\mathrm{x}$-axis, without acceleration, from the heater center $(\mathrm{x}=0)$ to the cavity boundary $(\mathrm{x}=570 \mu \mathrm{m})$ with $\mathrm{Th}=700 \mathrm{~K}$ $\mathrm{Ta}=300 \mathrm{~K}$. Comparison beetween FEM simulation and analytical result.

Figure 5 compares with very good agreement the temperature profile calculated along $\mathrm{x}$-axis using expression (4) with FEM simulation results. For $x=d=200 \mu \mathrm{m}$, i.e. at the location of detectors, the temperature is $153^{\circ} \mathrm{C}$ above ambient temperature.

\section{Section 3: Fluid Convection}

In the thermal accelerometer, the gas density lowers as its temperature grows. Therefore, an applied acceleration results in a gas movement, following Archimede's law. The temperature profile previously calculated deforms, producing a temperature difference across the detectors $\Delta \mathrm{T}_{\mathrm{D}}$. Interestingly, this temperature difference has been found to be proportional to the Grashof number determined for an acceleration along the sensitive axis [1]. It comes:

$\Delta T_{D}=S \cdot G r$ with $G r=\frac{a \cdot \rho^{2} \cdot(T s-T a) \cdot l^{3}}{\mu^{2}}$

with $\mathrm{S}(\mathrm{K})$ a fitting coefficient which represents the system sensitivity, $a\left(\mathrm{~m} . \mathrm{s}^{-2}\right)$ the acceleration, $\rho\left(\mathrm{kg} \cdot \mathrm{m}^{-3}\right)$ the gas density, $\beta\left(\mathrm{K}^{-1}\right)$ the gas coefficient of expansion in, $\mu$ $\left(\mathrm{kg} \cdot \mathrm{m}^{-1} \cdot \mathrm{s}^{-1}\right)$ the gas viscosity and $1(\mathrm{~m})$ a fitting dimension related to the cavity volume and shape.

The coefficient $\mathrm{S}$ can then be extracted from FEM analysis or experiment. Figure 6 plots the temperature variation along $\mathrm{X}$-axis when a $1 \mathrm{~g}$ acceleration is applied to the sensor leading to an estimation of $\Delta \mathrm{T}_{\mathrm{D}}=1.5^{\circ} \mathrm{C} / \mathrm{g}$ for the test vehicle A. 


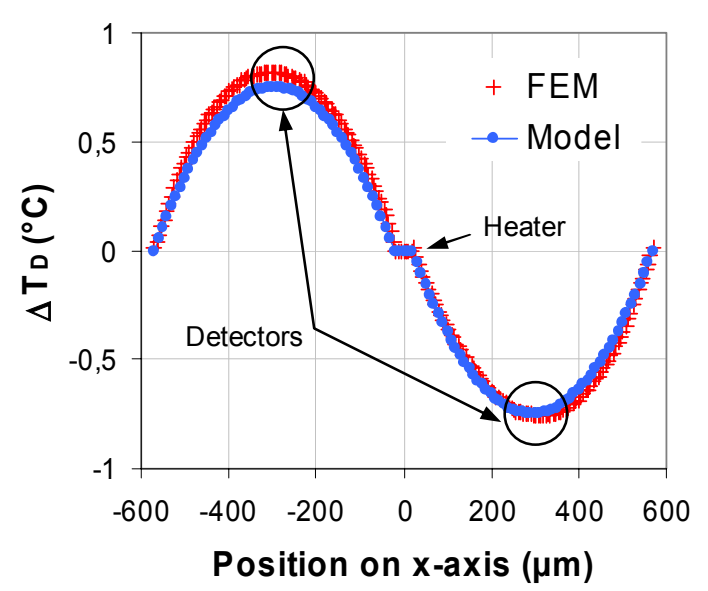

Figure 6. Temperature change along $\mathrm{x}$-axis for a $1 \mathrm{~g}$ acceleration. Comparison beetween FEM simulation and analytical result.

In addition, the profile of temperature variation obtained from FEM has been approximated by a polynomial function (see Fig. 6). This allows the HDL model to include the distance $d$ of the detectors from the heater as a design parameter, together with $r_{1}$ and $r_{2}$ dimensions:

$$
\Delta T(x)=\frac{2 \cdot \Delta T_{\max }}{(r 1-r 2) \cdot(r 2-r 1)} \cdot(x-r 1) \cdot(x-r 2) \quad(x \in[r 1 ; r 2])
$$

Obviously, the distance $d$ of the detector is optimized when $\Delta \mathrm{T}_{\mathrm{D}}=\Delta \mathrm{T}_{\text {max }}$. As a rule of the thumb, the detectors are best located midrange between the heater and the cavity boundary.

\section{Section 4: Sensing}

The detectors $R d_{1}$ and $R d_{2}$ convert the thermal signal into an electrical one. The model is simply based on the temperature sensitivity of the polysilicon:

$$
R d_{i}=R d_{i, n o m}\left(1+T C R \cdot \Delta T_{D, i}\right)
$$

A white noise spectral density is also implemented in the model for each detector in order to enable the simulation of the sensor performance in terms of resolution:

$$
V_{n, i}{ }^{2}(f)=4 \times k \times T_{D, i} \times R d_{i} \quad\left(\mathrm{~V}^{2} / \mathrm{Hz}\right)
$$

Where $k=1.38 \cdot 10^{-23} \mathrm{~J}^{-\mathrm{K}^{-1}}$ is the Boltzman's constant, and $T_{D, i}(\mathrm{~K})$ the temperature of each detector.

\section{E. Considerations for dynamic simulation}

For dynamic simulations of the heating bridge, it has been established that its thermal response corresponds to a first order low-pass system. A time constant of $\tau_{h}=3 \mathrm{~ms}$ has been measured on the test structure A, leading to an equivalent thermal capacity of about $C_{t h}=2.8 \cdot 10^{-7} \mathrm{~J} / \mathrm{K}$.

Concerning the dynamic response of the detector, we used the parameters extracted from the heater due to the relatively close geometry of the two types of bridges.

The dynamic response of the gas into the top and bottom cavities also corresponds to a first order low-pass response, has been established in [6]. Because the time constant strongly depends on package conditions (i.e. the cavities dimensions), the analytical modeling cannot be detailed here. On test-vehicle A, a response time of $30 \mathrm{~ms}$ has been estimated for the gas.

\section{CONCLUSION}

This paper concerns the modeling of a CMOS convective accelerometer for HDL integration. The sensor is divided into four functional blocs: (i) the heater; (ii) the gas conduction; (iii) the gas convection; and (iv) the detectors. For each bloc, an analytical model is proposed and validated using characterization or FEM simulation results. The influence of the top and bottom cavities have been previously studied using FEM but are not yet available in an analytical form.

\section{REFERENCES}

[1] A.M. Leung, J. Jones, E. Czyzewska, J. Chen, B. Woods, "Micromachined accelerometer based on convection heat transfer", proc. IEEE MEMS'98, Heidelberg, Germany, 25-29 Jan. 1998, pp. $627-630$.

[2] V. Milanovic, E. Bowen, M. E. Zaghloul, N. H. Tea , J. S. Suehle, B. Payne, and M. Gaitan, "Micromachined convective accelerometers in standard integrated circuits technology", Appl. Phys. Lett. 76 (4) (2000), pp. 508-510.

[3] X.B. Luo, Y.J. Yang, F. Zheng, Z.X. Li and Z.Y. Guo, "An optimized micromachined convective accelerometer with no proof mass", J. Michromech. Microeng. 11 (2001), pp. 504-508.

[4] F. Mailly, A. Giani, A. Martinez, R. Bonnot, P. Temple-Boyer and A. Boyer, "Micromachined thermal accelerometer", Sens. Actuators A 103 (3) (2003), pp. 359-363.

[5] See http://www.memsic.com

[6] A. Chaehoi, L. Latorre, F. Mailly, P. Nouet, "Experimental and Finite Element Study of Convective Accelerometer on CMOS"; proc. EUROSENSORS'05, Barcelona, Spain, Sept. 11-14, 2005. 